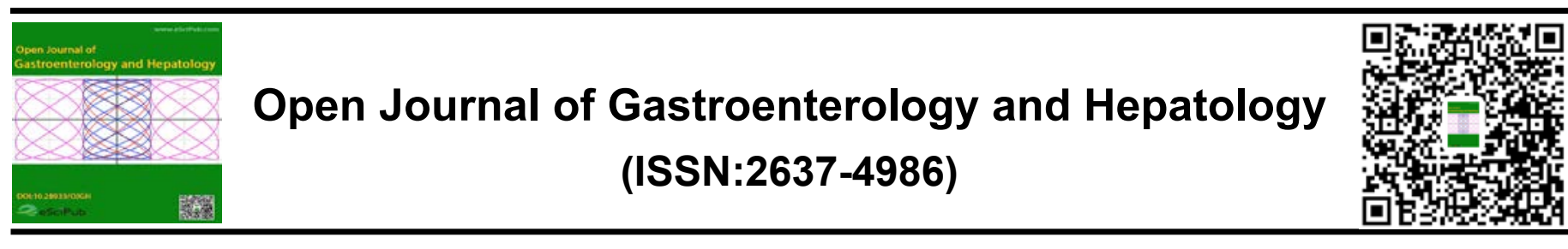

\title{
Effectiveness and safety of glecaprevir and pibrentasvir for hemodialysis patients with hepatitis $C$ virus infection at a single
} center

\section{Naoki Hotta}

Department of Hepatology, Masuko Memorial Hospital

\section{ABSTRACT}

Background/Aims: Glecaprevir/pibrentasvir (GLE/PIB) is a pan-genotypic regimen for the treatment of hepatitis $B$ virus (HCV) infection. GLE and PIB are direct acting antiviral (DAA) agents that can be used for patients with chronic renal failure who are on hemodialysis (HD) and those with HCV genotype 2 infections. Here, we report the usefulness and safety of GLE/PIB in 13 hemodialysis (HD) patients with HCV infection.

Material and Methods: The subjects comprised patients with genotype 1 and 2 (six each) and one unknown genotype patient in whom GLE/PIB therapy was introduced by December 2018. The mean age was 69.2 (59-78) years (seven men and six women). The mean HCV RNA amount prior to treatment initiation was 4.81 (2.1-6.5). The administration periods were 8 and 12 weeks ( $\mathrm{n}$ $=9$ and 4 , respectively).

Results: Twelve patients received all the doses orally while an increase in total bilirubin (T-BIL) caused administration to be discontinued in one patient. HCV RNA at week 4 after treatment initiation became undetectable in $11(91.6 \%)$ of the 12 patients.

*Correspondence to Author:

Naoki Hotta

Departmernt of Hepatology, Masuko Memorial Hospital

Email: hotta4166@yahoo.co.jp

How to cite this article:

Naoki Hotta.Effectiveness and safety of glecaprevir and pibrentasvir for hemodialysis patients with hepatitis $C$ virus infection at a single center. Open Journal of Gastroenterology and Hepatology, 2019, 2:26 All patients achieved rapid viral response (RVR). Concerning adverse effects, although itching occurred in three (25\%) patients, the symptom improved following administration of oral medication and the treatment was able to be continued.

Conclusion: The results suggest that GLE/PIB can also be safely administered to HD patients. However, the usefulness and safety need to be further studied by examining more cases.

Keywords: hepatitis $\mathrm{C}$, hemodialysis,

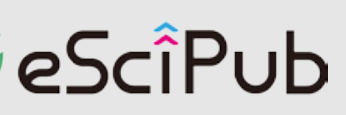

eSciPub LLC, Houston, TX USA. Website: https://escipub.com/ 


\section{Introduction}

The treatment of chronic hepatitis $\mathrm{C}(\mathrm{HCV})$ with interferon (INF) started in 1992 and the complete response rate was low. However, with the concomitant use of oral medications, which started in 2016, the complete response rate has increased to over $90 \%$. Various medications for genotype 1-infected patients on hemodialysis (HD) have emerged and the complete response rate has remarkably improved, whereas there are no medications for genotype 2-infected patients on HD. Glecaprevir/ pibrentasvir (GLE/PIB) is a pan-genotypic regimen for the treatment of HCV infection. GLE and PIB are direct-acting antiviral (DAA) agents that can be used for patients with chronic renal failure who are on HD and in genotype2-infected patients. Here, we report the usefulness and safety of this regimen for hemodialysis (HD) patients.

\section{Materials and Methods}

\section{Patient characteristics}

The subjects included in this study were 13 patients in whom GLE/PIB therapy was introduced by December 2018, consisting of six patients each with genotype 1 and 2 infection and one with unknown genotype infection. The mean age was 69.2 (59-78) years (seven men and six women). The mean HCV RNA amount was 4.81 (2.1-6.5) and the administration periods were 8 and 12 weeks ( $\mathrm{n}=9$ and 4 , respectively, Table 1).

No patient had a medical history of hepatocellular carcinoma (HCC) in the present study and this was the first viral therapy for all the patients. We explained the objectives and methods in writing to subjects and obtained informed consent. The study was conducted in accordance with the principles of the Declaration of Helsinki following the ethical guidelines for medical research involving human subjects (The Ministry of Education, Science, Sports and Culture and The Ministry of Health, Labor and Welfare, revised in 2014) and received approval from the ethics committee to perform the present study.

\section{Results}

Twelve patients received all the doses orally while elevation of the total bilirubin (T-Bil) value to $5.0 \mathrm{mg} / \mathrm{dL}$ in one patient on day 4 of administration, led to treatment discontinuation at the request of the patient and the patient's family. The T-Bil value improved to the normal level after discontinuation. HCV RNA at week 4 after treatment initiation became undetectable in $11(91.6 \%)$ of the 12 patients. It was also undetectable by week 8 in 11 patients and the sustained virologic response rate (SVR) was $100 \%$ by week 12 after treatment completion (Fig 1).

Although itching occurred in two (33\%) patients, the symptom improved following oral administration of medication and the study treatment was continued.

\section{Discussion}

HD patients with chronic renal failure are at numerous risks for exposure to HCV such as during administration of blood products, invasive dialysis operation, and frequent hospital visit. The number of deaths of HD patients related to liver disease is 5.89 -fold higher in those infected 
Table 1. Patient characteristics

\begin{tabular}{ll}
\hline Sex ratio (men/women) & $7 / 6$ \\
\hline Age (years) & $69.2(59-78)$ \\
White blood cells & $9861(2700-6800)$ \\
Hemoglobin & $10.7(9.3-14.2)$ \\
Platelets & $13.9(8.9-20.7)$ \\
AST & $20(11-41)$ \\
ALT & $14(6-33)$ \\
Serum albumin & $3.62(2.8-4.1)$ \\
FIB-4 index(<3.25/3.25æ) & $2 / 11$ \\
Wisreria floribunda agglutinin + Mac-2-binding protein (C.O.I) & $2.26(0.53-6.38)$ \\
Alpha-fetoprotein(ng/mL) & $2.85(1.17-5.52)$ \\
HCV RNA (log IU/mL) & $4.81(2.1-6.5)$ \\
Serotype(1/2/non) & $6 / 6 / 1$ \\
A history of treatment & None \\
Administration period & $(8 / 12$ week)9/4 \\
\hline
\end{tabular}

AST, aspartate transaminase; ALT, alanine aminotransaminase; FIB-4, fibrosis index based on the four factors

8

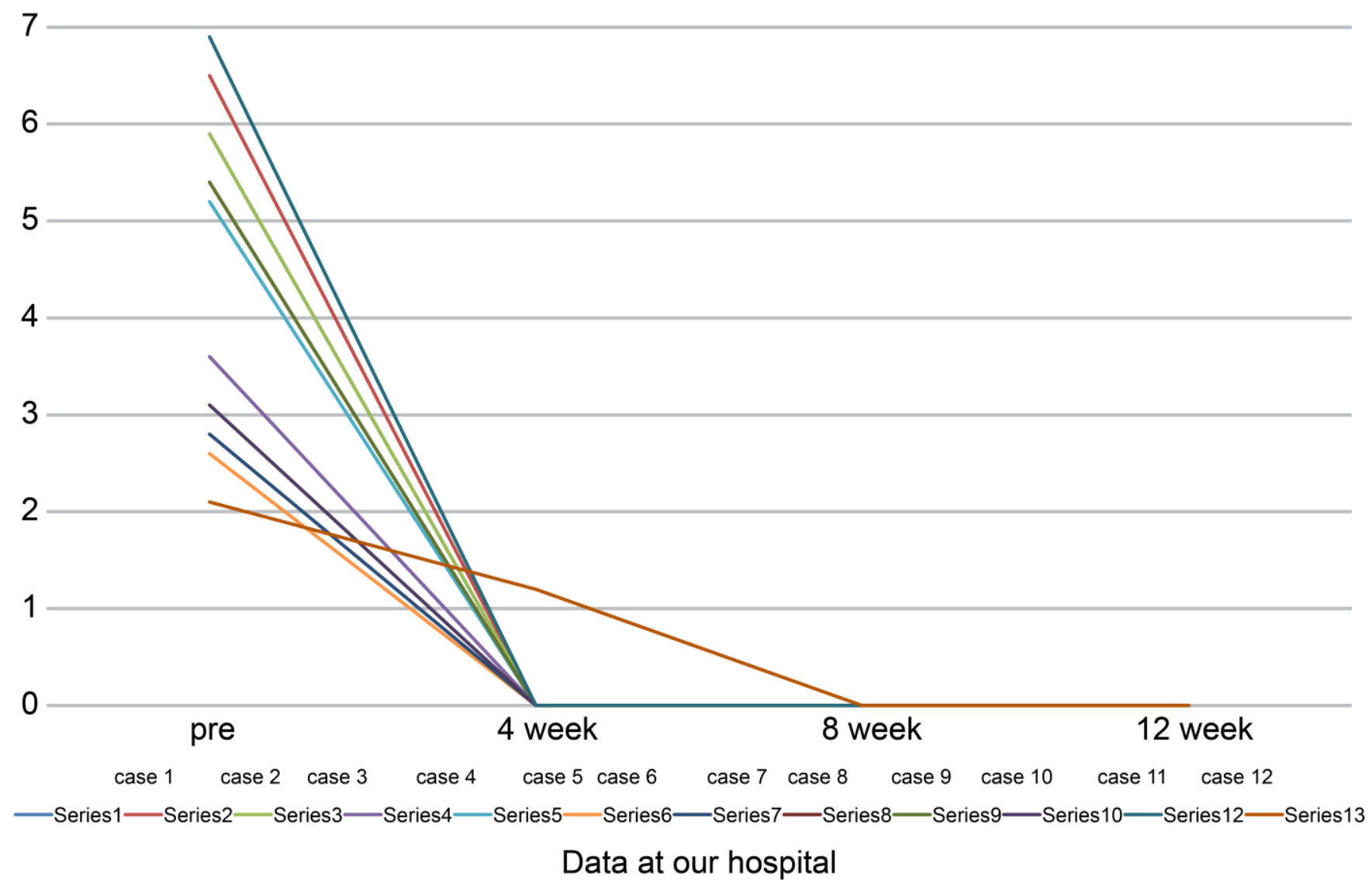

Fig 1 Changes in quantity of virus after treatment

OJGH: https://escipub.com/open-journal-of-gastroenterology-and-hepatology/ 
Table 2. Pretreatment blood test results (63-year-old woman)

\begin{tabular}{|c|c|}
\hline \multicolumn{2}{|c|}{ Peripheral blood } \\
\hline WBC & $5500 / \mu \mathrm{L}$ \\
\hline $\mathrm{RBC}$ & $386 \times 10^{4} / \mu \mathrm{L}$ \\
\hline $\mathrm{Hb}$ & $13.2 \mathrm{~g} / \mathrm{dL}$ \\
\hline Plt & $15.8 \times 10^{4} / \mu \mathrm{L}$ \\
\hline M2BPGi & 2.27 \\
\hline P-3-P & $49.4 \mathrm{ng} / \mathrm{mL}$ \\
\hline Type IV C.7S & $5.3 \mathrm{ng} / \mathrm{mL}$ \\
\hline AFP & $2.4 \mathrm{ng} / \mathrm{mL}$ \\
\hline Fib 4-index & 1.68 \\
\hline Age & 63 \\
\hline AST & 14 \\
\hline ALT & 11 \\
\hline PLT & 15.8 \\
\hline \multicolumn{2}{|c|}{ Blood chemistry } \\
\hline AST & $14 \mathrm{IU} / \mathrm{L}$ \\
\hline ALT & $11 \mathrm{IU} / \mathrm{L}$ \\
\hline $\mathrm{LDH}$ & $207 \mathrm{IU} / \mathrm{L}$ \\
\hline ALP & $275 \mathrm{IU} / \mathrm{L}$ \\
\hline v-GTP & $19 \mathrm{U} / \mathrm{L}$ \\
\hline TP & $5.7 \mathrm{~g} / \mathrm{dL}$ \\
\hline Alb & $3.6 \mathrm{~g} / \mathrm{dL}$ \\
\hline BUN & $46 \mathrm{mg} / \mathrm{dL}$ \\
\hline $\mathrm{Cr}$ & $7.77 \mathrm{mg} / \mathrm{dL}$ \\
\hline GFR & 5 \\
\hline \multicolumn{2}{|l|}{ Viral marker } \\
\hline HBs-Ag & negative \\
\hline HCV group & 2 \\
\hline HCV-RNA & 7.2 \\
\hline
\end{tabular}


with HCV than uninfected patients and been high enough and the subjects were limited to shown to be caused by the development of young patients and those with less cirrhosis and liver cancer (1). The study of INF complications (2). Then, the administration of therapy for HD patients by Kikuchi et al. (2) INF-free oral medications commenced in 2016 showed an SVR of $39 \%(22 / 56)$ in the REACH and was indicated for HD patients with genotype Study of PEG-INFa2a, in which the SVR was not $1 \mathrm{HCV}$ infections.

\section{5}

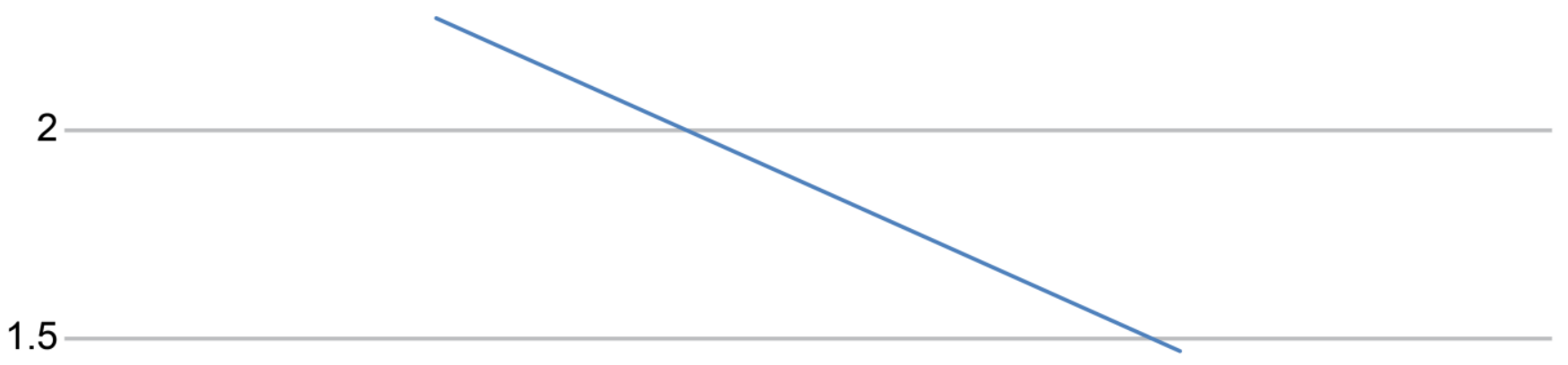

1

0.5

Prior to treatment

6 months after treatment

Fig 2 Changes in Mac-2-binding protein glycosylation isomers (M2BPGi) level before and after treatment

Toyoda et al. (3) and Suda et al. (4) reported that the SVR12 reached $100 \%(28 / 28)$ and $95.5 \%$ (20/21), respectively in HD patients. However, oral medications were not indicated for genotype 2 patients. Although the subsequently launched ombitasvir/ paritaprevir/ ritonavir combination was also indicated for HD patients, these agents were contraindicated for concomitant use with many medications and were not indicated for genotype 2 patients (5). The SVR was reported to be $99 \%$ following the use of grazoprevir and elbasuvir, which were also not indicated for genotype 2 patients (6). A pan-genotypic regimen of GLE/PIB, which is indicated for 
genotype $2 \mathrm{HD}$ patients is considered useful for the treatment of HD patients with complicated HCV infection (7-9).

We successfully administered the regimen for each indicated period to all six genotype 2 patients at our hospital. However, the period of oral administration needed to be set to 8 or 12 weeks in the present study. Based on the guidelines for HD indicate, it is difficult to compare the aspartate aminotransferase/ alanine aminotransferase (AST/ALT) values of HD patients with those of normal individuals. However, even when the liver function is normal, liver fibrosis is shown to be advanced. Presently, markers of liver fibrosis such as Mac-2-binding protein glycosylation isomers (M2BPGi) (10) and autotaxin have emerged. The fibrosis index based on the four factors (FIB4 -INDX), age (years) $\times$ AST $(U / L) /$ platelets $(109 / L) \times A L T(U / L)$ (11) is a serum marker of fibrosis. This is one of the markers of fibrosis based on age, AST, ALT, and platelets.

This marker only uses blood test results measured at each examination in ordinary clinical settings to calculate values using the formula. A value $\geq 3.25$ is known to indicate advanced liver fibrosis. The present study, which used this index showed no increase in the value in genotype 2 patients, whereas an increase was observed in two genotype 1 patients administered the regimen for 12 weeks and fibrosis was found. M2BPGi is a glycomarker that specifically detects a change in the sugar chain structure of M2BP caused by liver fibrosis. Since the value increases as liver fibrosis advances, this protein is useful as a marker that can predict the degree of advancement from chronic hepatitis to cirrhosis without performing a liver biopsy and, therefore, came to be covered by insurance in 2015. Our study showed that there was no change in M2BPGi before and after dialysis.

In the present study, the highest value was 6.38 (12). To develop a therapeutic strategy, liver fibrosis needs to be predicted using these markers and the administration period of oral medications should be set to 8 or 12 weeks. One case of a 63-year-old female patient with genotype $2 \mathrm{HCV}$ infection is presented below (Table 2). Based on the high values of the fibrosis markers, M2BPGi (2.27), and P-3-P (49.4 $\mathrm{ng} / \mathrm{mL})$, we decided to administer the regimen for 12 weeks. In the present study, the virus was eliminated from this patient in 8 weeks, and it did not flare up subsequently. In addition, although the M2BPGi level was 2.27 before treatment, it decreased to 1.476 months after treatment (Fig 2).

The results suggested that this treatment strategy improved liver fibrosis. Concerning adverse effects, itching, which was observed in three patients, did not appear immediately after administration but it occurred after week 2 of administration. The itching did not occur at the same time as drug administration and both patients were treated with medications. These patients were managed with nalfurafine hydrochloride, which improved the symptoms and allowed continuation of oral administration (13). Adverse effects may not occur immediately 
after administration as seen in these cases; thus, patients should always be examined at week 1 and 2 after administration to monitor the symptom to ensure the treatment is safely conducted.

In contrast, patients with no symptom can be followed during long-term administration at 1 or 2 weeks after initial administration. In the present study, one patient had an unknown genotype infection and exhibited viral elimination by week 12 of administration. The treatment for all patients in the present study was conducted at our hospital and the branch and patient information including blood test results were available in electronic medical records, which allowed prompt management. Moreover, the effectiveness and safety of the treatment has not been previously reported by a single facility.

Okubo et al. (14) conducted an epidemiological study of the incidence of HCV infection and the status of interventions in approximately 3,000 HD patients at 31 dialysis facilities. Their findings demonstrated that the HCV antibody-positive rate was $5.3 \%$, which was clearly higher than that of non-HD patients, and $45.5 \%$ of patients received interventions with anti-viral therapy and, thus, interventions were not performed in many patients (14). Administration of the medications investigated in the present study allowed the safe completion of treatment in 12 weeks at the most, which would contribute to the prevention of cirrhosis and HCC caused by advancement of liver fibrosis. Thus, we encourage patients to be aware of these facts when visiting hepatologists. Lastly, the results showed that these oral medications can be safely used without any serious adverse effects, providing benefits to genotype 2 patients. Following the launching of new oral medications for genotype 2, pangenotype treatment does not seem to be widely prescribed. However, patients should be treated with these medications based on the benefits, and therefore, it is very important to determine the state of liver fibrosis and establish an appropriate administration period. Although the number of cases was small in the present study, we plan to examine additional cases to further study the safety and effectiveness.

\section{Conclusion}

Our findings indicate that GLE/PIB are safe and effective DAA medications for HD patients as well. Finally, the usefulness and safety of this regimen needs to be further studied by accumulating and examining more patient cases.

\section{References}

1. Fabrizi F, Dixit V, Messa P, Martin P. Hepatitis Crelated liver diseases in dialysis patients. Contrib Nephrol 2012; 176: 42-53.

2. Kikuchi K, Akiba T, Nitta K, et al. Multicenter study of pegylated interferon $\alpha-2 a$ monotherapy for hepatitis C virus-infected patients on hemodialysis: REACH study. Ther Apher Dial 2014; 18: 603-11.

3. Toyoda H, Kumada T, Tada T, et al. Safety and efficacy of dual direct-acting antiviral therapy (daclatasvir and asunaprevir) for chronic hepatitis C virus genotype 1 infection in patients on hemodialysis. J Gastroenterol 2016; 51: 741-7.

4. Suda G, Kudo M, Nagasaka A, et al. Efficacy and 
safety of daclatasvir and asunaprevir combination therapy in chronic hemodialysis patients with chronic hepatitis C. J Gastroenterol 2016; 51: 733-40.

5. Atsukawa M, Tsubota A, et al. Efficacy and safety of ombitasvir/paritaprevir/ritonavir in dialysis patients with genotype $1 \mathrm{~b}$ chronic hepatitis C. Hepatol Res 2017; 47: 1429-36.

6. Edward G, Eric L, David P, et al. Glecaprevir and pibrentasvir in patients with $\mathrm{HCV}$ and severe renal impairment. N Engl J Med 2017; 12: 1448-55.

7. Hotta N. Efficacy and safety of glecaprevir/pibrentavir in combination therapy in chronic hemodialysis patients with genotype 2 hepatitis C virus infection.Open J Gastroenterol 2019; 9: 1-6.

8. Atsukawa M, Tsubota A, Toyoda $\mathrm{H}$, et al. The efficacy and safety of glecaprevir/pibrentavir in 141 patients with severe renal impairment: prospective, multicenter study. Aliment Pharmacol Ther 2019; 49: 1-12.

9. Toyoda H, Chyayama K, Suzuki F, et al. Efficacy and safety of glecaprevir/pibrentavir in Japanese patients with chronic genotype 2 hepatitis $C$ virus infection. Hepatology 2018; 67: 505-513.

10. Ura K, Furusyo N, Ogawa E, et al. Serum WFA(+) $-\mathrm{M} 2 \mathrm{BP}$ is a non-invasive liver fibrosis marker that can predict the efficacy of direct acting anti-viralbased triple therapy for chronic hepatitis $\mathrm{C}$. Aliment Pharmacol Ther 2016; 43: 114-24.

11. Shah AG, Lydecker A, Murray K, et al. Comparison of noninvasive markers of fibrosis in patients with nonalcoholic fatty liver disease. Clin Gastroenterol Hepatol 2009; 7: 1104-12.

12. Naoki H. M2BPGi Effects of dialysis. Sysmex Data 2017.

13. Kamimura $\mathrm{K}$, Yokoo $\mathrm{T}$, Kamimura $\mathrm{H}$, et al. Longterm efficacy and safety of nalfurafine hydrochloride on pruritus in chronic liver disease patients: Patient-reported outcome based analyses. PLoS One 2017; 12: 1-11.

14. Okuubo T, Atsukawa M, Tsubota $A$, et al. Epidemiological survey of patients with hemodialysis complicated by hepatitis $\mathrm{C}$ in Japan. Ther Apher Dial 2019; 23: 44-8.

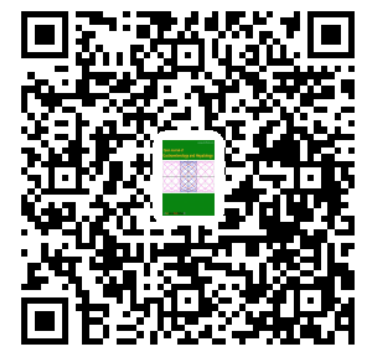

